\title{
Lansoprazole-Induced Subacute Cutaneous Lupus Erythematosus
}

\author{
İsa AN, ${ }^{1}$ Vasfiye DEMİR, ${ }^{2}$ İbrahim İBİLOĞLU, ${ }^{3}$ Sedat AKDENİZ, ${ }^{1}$ Derya UÇMAK ${ }^{1}$ \\ ${ }^{1}$ Department of Dermatology, Medical Faculty of Dicle University, Diyarbakır, Turkey \\ ${ }^{2}$ Department of Family Medicine, Medical Faculty of Dicle University, Diyarbakır, Turkey \\ ${ }^{3}$ Department of Pathology, Medical Faculty of Dicle University, Diyarbakır, Turkey
}

A 23-year-old female patient presented with a one-week history of itchy skin lesions distributed on the upper chest, extensor sides of the arms, and the trunk. Two weeks earlier, the patient had been prescribed lansoprazole $40 \mathrm{mg} /$ day due to the symptoms of gastroesophageal reflux. Physical examination revealed multiple erythematous, annular plaques of varying sizes distributed on the upper chest, arms and trunk (Figure 1). In laboratory analysis of the blood, antinuclear antibodies were detected at a titer of $1 / 160$ with a diffuse pattern. Anti-doublestranded deoxyribonucleic acid was negative and anti-Ro/Sjögren's syndrome A (SSA), anti-La/ Sjögren's syndrome B (SSB) antibodies, and antihistone antibodies were positive. Histopathologic examination of the skin biopsy revealed atrophic epidermis and vacuolar degeneration of the basal layer (Figures 2a, b). Immunofluorescence was weakly positive for $\mathrm{C} 3$ at the basement membrane. Depending on the clinical history, autoantibody profile, and the histopathologic findings, the patient was diagnosed as having lansoprazole-induced subacute cutaneous lupus erythematosus (SCLE). The lansoprazole therapy was discontinued and the patient was given hydroxychloroquine sulfate $200 \mathrm{mg} /$ day. At the follow-up, all lesions disappeared after one month. Hydroxychloroquine sulfate therapy was stopped after three months and it was observed that no lesions occurred during one-year follow-up. A written informed consent was obtained from the patient.

Subacute cutaneous lupus erythematosus is a subtype of lupus erythematosus, characterized by polycyclic annular erythematous plaques and limited systemic involvement. Drug induced subacute cutaneous lupus erythematosus (DI-SCLE) mostly affects adults and often occurs on sun-exposed areas. ${ }^{1}$ DI-SCLE has been shown to be associated with a large number of drugs including calcium channel blockers, statins, and proton pump inhibitors. ${ }^{2}$ Although the patients with autoimmune diseases have been shown to be more prone to DI-SCLE, the exact pathogenesis of DI-SCLE remains unknown. Anti-Ro/SSA

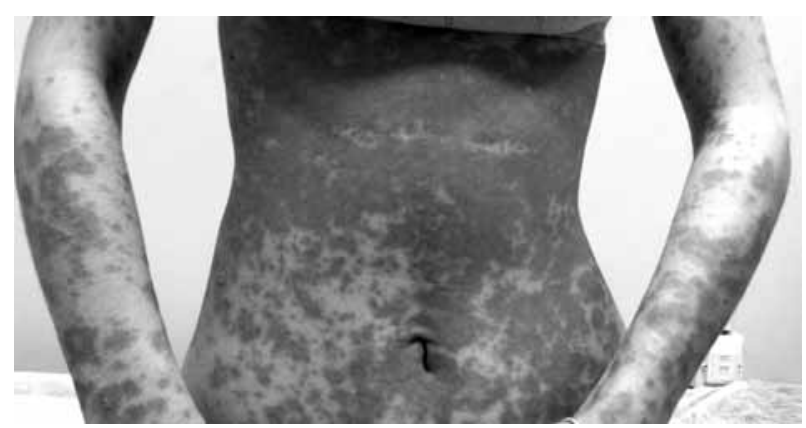

Figure 1. Multiple erythematous, squamous, annular plaques of varying sizes are seen on upper arms and trunk. 

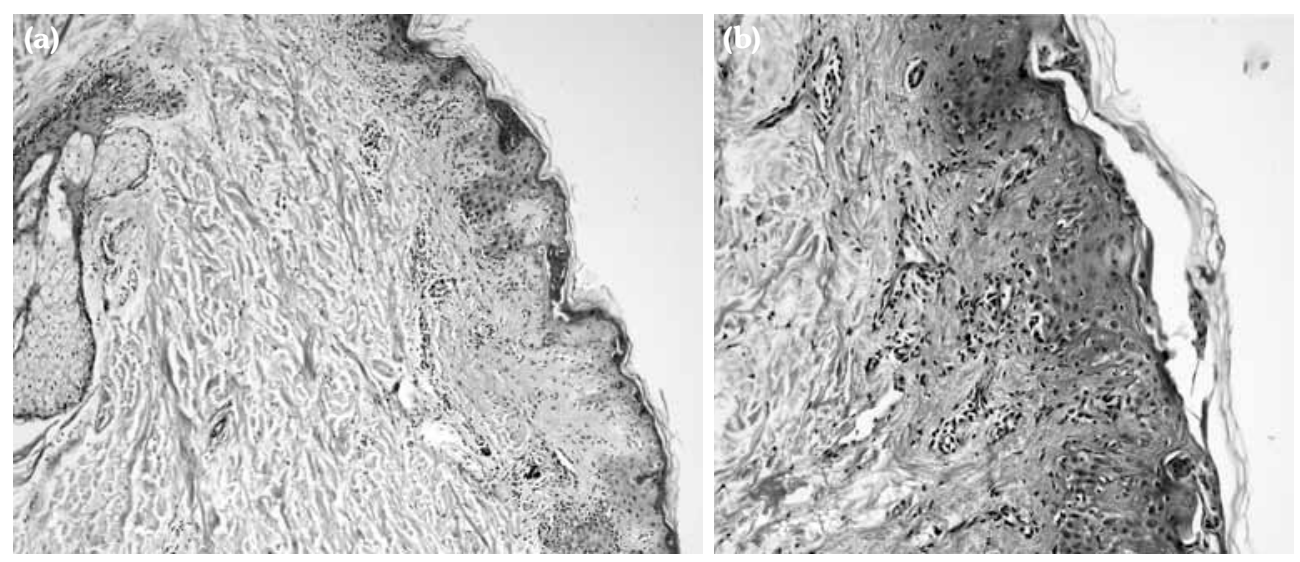

Figure 2. (a) Atrophic epidermis, vacuolar degeneration of basal layer, edema in upper dermis, and lymphocytic infiltration $(\mathrm{H}-\mathrm{E} \times 100)$. (b) Irregularity and patchy thickening of basement membrane (periodic acid shift $\times 200$ ).

antibodies are positive in $90 \%$ of the patients with DI-SCLE. However, positive anti-La/SSB antibodies are found in a very small number of patients, and anti-histone antibodies are positive in only one third of the patients. ${ }^{2,3}$

Although DI-SCLE has been shown to cause more disseminated cutaneous manifestations and a higher incidence of vasculitic and bullous lesions, DI-SCLE cannot be easily differentiated from idiopathic SCLE clinically, histopathologically, or immunologically. In patients with DI-SCLE, the histological examination of a skin biopsy often indicates vacuolar degeneration of the epidermal basal layer, necrotic keratinocytes, and lymphocytic interface dermatitis. ${ }^{4}$ DI-SCLE commonly occurs between three days and 11 years after the introduction of the causative drug. Considering that the skin lesions resolve after the withdrawal of the causative drug, the withdrawal of the drug is the cornerstone for the management of DI-SCLE. ${ }^{2,3}$ Lansoprazole is a potent proton pump inhibitor used for the treatment of peptic ulcer. Cutaneous side effects of lansoprazole are lichen planus, urticaria, and toxic epidermal necrolysis., ${ }^{1,5}$ Lansoprazoleinduced SCLE has been reported in very few studies. $^{1-3}$

In patients presenting with lupus erythematosus, clinicians should obtain a detailed drug history of the patients and should keep in mind that lansoprazole, which is a frequently prescribed drug, may cause DI-SCLE, though rarely.

\section{Declaration of conflicting interests}

The authors declared no conflicts of interest with respect to the authorship and/or publication of this article.

\section{Funding}

The authors received no financial support for the research and/or authorship of this article.

\section{REFERENCES}

1. Sandholdt LH, Laurinaviciene R, Bygum A. Proton pump inhibitor-induced subacute cutaneous lupus erythematosus. Br J Dermatol 2014;170:342-51.

2. Pretel M, Marquès L, España A. Drug-induced lupus erythematosus. Actas Dermosifiliogr 2014;105:18-30.

3. Lowe GC, Henderson CL, Grau RH, Hansen CB, Sontheimer RD. A systematic review of drug-induced subacute cutaneous lupus erythematosus. Br JDermatol 2011;164:465-72.

4. Sontheimer RD. Subacute cutaneous lupus erythematosus: 25-year evolution of a prototypic subset (subphenotype) of lupus erythematosus defined by characteristic cutaneous, pathological, immunological, and genetic findings. Autoimmun Rev 2005;4:253-63.

5. Chang YS. Hypersensitivity reactions to proton pump inhibitors. Curr Opin Allergy Clin Immunol 2012;12:348-53. 